\title{
Desenvolvimento de um questionário quantitativo de freqüência alimentar em imigrantes japoneses e seus descendentes residentes em São Paulo, Brasil
}

\author{
Development of a dietary assessment \\ method for people of J apanese descent \\ living in São Paulo, Brazil
}

Marly Augusto Cardoso 1,2

Priscila Regina Stocco 1

\footnotetext{
1 Núcleo de Investigação em Nutrição, Instituto de Saúde. Rua Santo Antônio 590, Bela Vista, São Paulo, SP 01314-000, Brasil.

2 Departamento de Epidemiologia e Saúde Coletiva, Faculdade de Medicina de São José do Rio Preto. São José do Rio Preto, SP 15090-000, Brasil. marlyac@hotmail.com
}

Abstract The present study describes the devel opment of a questionnai re to assess usual frequency and quantity of food and nutrient intake by people of Japanese descent living in São Paulo, Brazil. Both the food list and appropriate serving sizes for food items from the Food Frequency Questionnaire (FFQ) were sel ected from a cross-sectional assessment of diet in a random sample of Japanese immigrants living in the city of São Paulo (first- and second-generations, $\mathrm{n}=166$ ), aged $45-70$ years, using self-administered three-day food records. We used the food records to sel ect food items on the basis of their contribution to total population intake of relevant foods and nutrients. Criteria for grouping separate foods included similarity in nutrient content per usual serving and the importance of a particular food (e.g., typical Japanese foods). Four portion sizes were presented: small, medium, large, and extra-large. We examined the frequency distribution of equival ent-gram weights of each sel ected food and identified the four serving sizes in the distribution. The quantitative FFQ was designed with 129 food groups and exact frequencies of intake. Instructions for self-reporting and applications of the method for epidemiological use are discussed.

Key words Food Consumption; Nutrition; Methods; Epidemiology

Resumo Descreve-se o desenvolvimento de um questionário para avaliação da freqüência habitual e da quanti dade de alimentos e nutrientes consumidos por indi víduos de etnia japonesa residentes em São Paulo. A lista de alimentos e preparações do Questionário Quantitativo de Freqüência Alimentar (QQFA) foi elaborada a partir de inquérito com registro de consumo alimentar de três dias em amostra aleatória de japoneses de primei ra e segunda geração da cidade deSão Paulo ( $n=166$; faixa etária 45 a 70 anos). Os alimentos foram agrupados segundo valor nutricional por porção al imentar e fontes de nutrientes de parti cular interesse (por exemplo, alimentos e preparações de origem japonesa). O tamanho das porções de cada item alimentar foi classificado em pequeno, médi o, grande extra grande de acordo com a distribui ção percentual dos pesos correspondentes às medi das casei ras referi das nos registros ali mentares. O QQFA foi elaborado com 129 itens al imentares e com perguntas a respei to de práticas e preferências alimentares usuais. O QQFA poderá ser auto-administrado em indivíduos de escolaridade de nível médi o ou superior. O tempo médio de preenchimento é de 45 mi nutos, dependendo da variabilidade da dieta habitual, faixa etária e moti vação do participante.

Palavras-chave Consumo de Alimentos; Nutrição; Métodos; Epidemiologia 
Introdução

Estudos prospectivos e de casos e controles têm fornecido evidências da importância da dieta na identificação de fatores de risco para doenças cardiovasculares, acidente vascular cerebral, diabetes e vários tipos de neoplasias malignas. As correlações mais consistentes provêm de comparações entre populações que se deslocaram para regiões com diferentes hábitos alimentares, diversa incidência de doenças crônicas e perfil de mortalidade distinto em relação ao país de origem (Haenszel \& Kurihara, 1968; Benfante et al., 1994; Cardoso et al., 1997).

Para identificar fatores de risco dietéticos em grupos populacionais no Brasil há necessidade de informações confiáveis quanto ao consumo alimentar habitual e ao teor de vários nutrientes em alimentos e preparações al imentares.

Investigações epidemiológicas relativas à dieta e a doenças crônicas exigem a utilização de metodologia padronizada para avaliação do consumo alimentar individual em grandes amostras populacionais mediante o emprego de instrumentos válidos, precisos e viáveis economicamente (Lee et al., 1983; Thompson \& Byers, 1994; Willett, 1998).

Entre os métodos de estimativa de consumo alimentar, o inquérito recordatório de 24 horas é provavelmente o mais utilizado na avaliação nutricional de populações no Brasil (Olinto et al., 1995). Esse método é de fácil aplicação e adequado à descrição de médias ou percentis de consumo al imentar de grupos de indivíduos (Thompson \& Byers, 1994). No entanto, um único inquérito recordatório de 24 horas não estima a dieta habitual individual, pois não considera a variabilidade do consumo alimentar de um dia para outro (intra-indivíduo). A variação intra-indivíduo e entre indivíduos pode também diferir muito entre nutrientes (McGee et al., 1982). Para nutrientes com pequena variação no consumo intra e interindivíduos - tais como carboidratos e gordura total - alguns dias de registro de consumo al imentar são suficientes para estimar sua ingestão média. No entanto, para outros nutrientes, como a vitamina A, a variação intra-individual é muito maior que a variabilidade interindividual, exigindo vários dias de registros alimentares para se atingir estimativa mais acurada do consumo real desses nutrientes (Nelson et al., 1989; Liu, 1994).

Para caracterizar a dieta habitual individual em pesquisa epidemiológica são necessários instrumentos de avaliação de consumo alimentar de fácil utilização e de baixo custo (Willett, 1994). Para atender a essas exigências, questio- nários quantitativos de freqüência alimentar (QQFA) têm sido empregados em estudos prospectivos internacionais (Willett et al., 1985; Block et al., 1986; Hankin \& Wilkens, 1994).

As etapas iniciais de desenvolvimento de um QQFA para inquéritos epidemiológicos incluem: a) seleção dos alimentos de acordo com o padrão dietético da população de estudo e b) identificação de porções alimentares adequadas às quantidades habitualmente consumidas pelos indivíduos da pesquisa (Hankin, 1986). O presente trabalho descreve o desenvolvimento de um QQFA para avaliação da dieta habitual individual - definida como ingestão alimentar usual no período de um ano - a ser utilizado em estudos epidemiológicos com imigrantes japoneses e seus descendentes residentes em São Paulo. A utilização de métodos de estimativa de consumo alimentar adequados e adaptados aos padrões dietéticos de populações migrantes e seus descendentes é essencial para a investigação do papel da dieta na etiologia de doenças crônicas (Hankin \& Wilkens, 1994).

\section{Materiais e métodos}

\section{População de estudo}

A lista de alimentos e preparações do QQFA foi elaborada a partir de um inquérito com registro de consumo alimentar de três dias em amostra aleatória de japoneses de primeira e segunda geração da cidade de São Paulo ( $n=166$; faixa etária $=45$ a 70 anos). Utilizou-se a população de estudo de inquéritos relativos a condições de saúde e estilo de vida de imigrantes japoneses residentes na cidade de São Paulo, descrita em publicações anteriores (Tsugane et al., 1994; Cardoso et al., 1997).

O universo amostral foi selecionado a partir de censo especial para a população japonesa no Brasil (Center for Japan-Brazil Studies, 1988). Foram identificados 273 domicílios com pelo menos um morador de origem japonesa em 264 áreas geográficas al eatoriamente escoIhidas. Nestes domicílios foram localizados os imigrantes japoneses (primeira geração) e descendentes (segunda geração) com idade entre 40 e 69 anos (em junho de 1989).

O total de indivíduos selecionados na amostra inicial foi de 411. Em 1989, 251 indivíduos (61\% do total) participaram do primeiro inquérito transversal a respeito das condições de saúde e estilo de vida. Em 1995, 166 participantes (40,4\% da amostra original), 78 homens e 89 mulheres, participaram de um segundo inquérito de saúde e completaram, nesta oca- 
sião, o registro de consumo alimentar de três dias. Embora esses resultados não representem o consumo alimentar da amostra populacional inicial, as informações desses registros foram tomadas em conta no presente estudo para construção da versão inicial do QQFA.

\section{Seleção dos alimentos do Q QFA}

As quantidades de alimentos referidas no registro de consumo alimentar foram expressas em medidas casei ras e convertidas em gramas ou ml. com auxílio do programa de computador Apoio à Nutrição (versão 2.5), desenvolvido pelo Centro de Informática em Saúde da Universidade Federal de São Paulo. Para complementação dessas informações foram utilizadas pesagem direta de alimentos e preparações em alguns domicílios. O cálculo da composição de nutrientes dos registros de consumo alimentar empregou o mesmo programa de computador com dados adicionais das tabelas oficiais do Brasil (IBGE, 1985) e do Japão (Resources Council, Science and Technology Agency, 1993). Detalhes acerca dos procedimentos adotados na coleta e análise nutricional dos registros de consumo alimentar encontram-se em publicação anterior (Cardoso et al., 1997).

A partir da análise nutricional dos registros de consumo alimentar, foram identificados 230 alimentos e bebidas responsáveis por $90 \%$ das calorias totais da população estudada. Esses itens foram então organizados em 129 grupos de al imentos. Os critérios para inclusão ou exclusão de alimentos em mesmo grupo consideraram a similaridade do valor nutricional por porção alimentar usual (e não por 100 gramas de alimento) e a importância de al guns al imentos na epidemiologia de doenças crônicas (por exemplo, derivados da soja e chá verde). Incluíram-se também outras questões referentes a práticas e preferências al imentares habituais, tais como, tipo de adoçante em bebidas, tipo de gordura utilizada no preparo das refeições, número de refeições por dia, ingestão de gordura visível de carnes, outros alimentos não listados de consumo semanal e uso de suplementos dietéticos.

\section{Cálculo da porção alimentar de referência}

O tamanho das porções de cada item al imentar do QQFA foi classificado em pequeno, médio (= porção de referência), grande e extra grande de acordo com a distribuição percentual dos pesos equivalentes às medidas caseiras dos registros alimentares. A porção mediana foi utilizada como referência em cada item do QQFA, atribuindo-se aos tamanhos pequeno, grande e extra grande os pesos dos percentis 25, 75 e 100, respectivamente. Este procedimento permite estimativa mais acurada do teor de nutrientes em questionários quantitativos de freqüência alimentar (Block et al., 1986).

\section{Resultados}

A Tabela 1 apresenta a lista de alimentos do QQFA. A composição dos grupos alimentares procurou garantir a avaliação adequada da ingestão de fibras e micronutrientes da dieta incluindo grande variedade de vegetais folhudos, frutas e legumes agrupados em vários itens. Foram incluídos também: alimentos associados a fatores adversos à saúde - por exemplo, conservas de vegetais, cogumelos, café, chá preto, adoçantes artificiais -; preparações regionais e alimentos étnicos - feijoada, estrogonofe, natto, manju - e alimentos cuja omissão poderia comprometer a classificação dos níveis de consumo de poucas pessoas que habitual mente os consomem - por exemplo, fígado bovino.

A Tabela 2 mostra a distribuição em percentis dos pesos das porções dos alimentos e preparações de origem japonesa incluídas no QQFA. A análise inicial da distribuição dos pesos das porções de todos os itens alimentares do questionário foi realizada segundo sexo. No entanto, observou-se que os valores eram semelhantes na maioria dos itens para a porção mediana, diferindo poucas vezes no percentil 25 - valores menores para mulheres - e percentil 100 - mai ores entre os homens -, adotandose então os valores encontrados na distribuição percentual geral da população estudada.

Observa-se, na Figura 1, o modelo adotado para o QQFA. Este desenho foi elaborado com base em dois questionários atualmente em uso em coortes internacionais no Japão (Tsubono et al., 1996) e em imigrantes do Havaí (Hankin, 1986), com questões abertas referentes à freqüência de consumo alimentar. O QQFA poderá ser adaptado para leitura óptica, a exemplo dos questionários internacionais. Para cada item alimentar do QQFA, os participantes registram a freqüência média usual de consumo de cada item, a respectiva unidade de tempo se diariamente, semanalmente, mensalmente ou anual mente - e qual o tamanho da porção individual usual - se pequena, média, grande ou extra grande em relação à porção de referência de cada item do QQFA.

Esta versão inicial do QQFA foi utilizada em estudo piloto relativo a hábitos alimentares e incidência de pólipos adenomatosos em indi- 
Tabela 1

Lista de alimentos do questionário quantitativo de freqüência alimentar.

\begin{tabular}{|c|c|c|c|}
\hline $\begin{array}{l}\text { sopas (de legumes, canja, } \\
\text { cremes etc.) }\end{array}$ & $\begin{array}{l}\text { Shiruko, zenzai (doce de feijão } \\
\text { adzuki) }\end{array}$ & brócolos, couve-flor & manteiga \\
\hline misoshiro & $\begin{array}{l}\text { lentilha, grão de bico } \\
\text { ou feijão branco }\end{array}$ & cenoura crua ou cozida & $\begin{array}{l}\text { requeijão, queijo cremoso } \\
\text { (comum, light) }\end{array}$ \\
\hline macarronada, ravióli etc. & soja (em grão) & $\begin{array}{l}\text { berinjela, chuchu, abobrinha } \\
\text { (refogado) }\end{array}$ & geléia ou mel \\
\hline lasanha ou caneloni & tofu fresco ou yakidofu & $\begin{array}{l}\text { pepino, pimentão, cru como } \\
\text { salada }\end{array}$ & $\begin{array}{l}\text { pasta de amendoim } \\
\text { ou patês }\end{array}$ \\
\hline pizza & natto & $\begin{array}{l}\text { milho verde (fresco, congelado } \\
\text { ou enlatado) }\end{array}$ & chocolates, brigadeiro \\
\hline yakisoba & leite de soja (tonyu) & $\begin{array}{l}\text { ervilha, vagem (fresco } \\
\text { ou enlatado) }\end{array}$ & $\begin{array}{l}\text { bolo, tortas, bombas, } \\
\text { pavês }\end{array}$ \\
\hline udon, soba, lamen & $\begin{array}{l}\text { miso (tempero de pratos que } \\
\text { não misoshiro) }\end{array}$ & $\begin{array}{l}\text { broto de feijão, de bambu } \\
\text { ou soja }\end{array}$ & sorvetes \\
\hline pastel, guioza & $\begin{array}{l}\text { ovos (omelete, cozido, } \\
\text { cru ou mexido) }\end{array}$ & nabo (daikon), bardana (gobó) & $\begin{array}{l}\text { pudins, flans, curau, } \\
\text { arroz doce }\end{array}$ \\
\hline $\begin{array}{l}\text { pastelaria salgada } \\
\text { (torta, empada, esfiha) }\end{array}$ & $\begin{array}{l}\text { arroz branco cozido } \\
\text { com óleo e temperos }\end{array}$ & $\begin{array}{l}\text { tempura de cenoura, } \\
\text { berinjela, cebola }\end{array}$ & $\begin{array}{l}\text { yookan, doce de abóbora } \\
\text { ou goiabada }\end{array}$ \\
\hline $\begin{array}{l}\text { estrogonofe de carne } \\
\text { e kare-raisu }\end{array}$ & arroz japonês sem óleo & $\begin{array}{l}\text { laranja, mexerica, tangerina } \\
\text { ou mikan }\end{array}$ & manju, daifuku \\
\hline $\begin{array}{l}\text { carne com vegetais (chop suey, } \\
\text { sukiyaki) }\end{array}$ & sushi, norimaki, makisushi & banana & amendoim, castanha de caju \\
\hline $\begin{array}{l}\text { chop suey de frango, frango } \\
\text { xadrez, nishime }\end{array}$ & batata frita ou mandioca frita & mamão & castanha do pará \\
\hline hambúrguer com pão & $\begin{array}{l}\text { batata ou mandioca assada } \\
\text { ou cozida }\end{array}$ & maçã, pêra & pipoca \\
\hline bife bovino frito & $\begin{array}{l}\text { purê de batata ou suflês, } \\
\text { salada de batata }\end{array}$ & caqui (na estação) & $\begin{array}{l}\text { salgadinhos, chips, } \\
\text { torresmo/pururuca }\end{array}$ \\
\hline $\begin{array}{l}\text { carne assada ou grelhada, } \\
\text { churrasco }\end{array}$ & batata doce ou abóbora & melancia & $\begin{array}{l}\text { refrigerantes (comum, } \\
\text { dietéticos) }\end{array}$ \\
\hline carne cozida picada ou moída & konnyaku & manga (na estação) & sucos artificiais \\
\hline $\begin{array}{l}\text { bife à milanesa } \\
\text { ou à parmegiana }\end{array}$ & $\begin{array}{l}\text { leite com café (integral, } \\
\text { desnatado) }\end{array}$ & melão, abacaxi & $\begin{array}{l}\text { tempero de saladas: } \\
\text { óleo, azeite de oliva } \\
\text { vinagrete, catchup/mostarda, }\end{array}$ \\
\hline embutidos (lingüiça, salsicha) & $\begin{array}{l}\text { leite sem café (integral, } \\
\text { desnatado) }\end{array}$ & kiwi, goiaba & maionese \\
\hline $\begin{array}{l}\text { presunto, mortadela, } \\
\text { outros frios }\end{array}$ & $\begin{array}{l}\text { chocolate, nescau, toddy } \\
\text { - adicionado ao leite }\end{array}$ & $\begin{array}{l}\text { uva, morango, pêssego, } \\
\text { ameixa }\end{array}$ & \\
\hline carne de porco & $\begin{array}{l}\text { neston, aveia, granola } \\
\text { em leite ou frutas }\end{array}$ & abacate & $\begin{array}{l}\text { bebidas alcóolicas: } \\
\text { cerveja, sake, pinga, } \\
\text { uísque ou vodca }\end{array}$ \\
\hline $\begin{array}{l}\text { frango à milanesa, } \\
\text { à dorê, nuggets }\end{array}$ & yakult & salada de frutas & conhaque, vinho \\
\hline $\begin{array}{l}\text { frango assado ou grelhado, } \\
\text { espeto }\end{array}$ & $\begin{array}{l}\text { iogurte (integral, com frutas, } \\
\text { desnatado, light) }\end{array}$ & frutas em calda & \\
\hline $\begin{array}{l}\text { miúdos de frango (coração, } \\
\text { moela, fígado) }\end{array}$ & vitamina de leite & suco de laranja natural & \\
\hline
\end{tabular}


Tabela 1 (continuação)

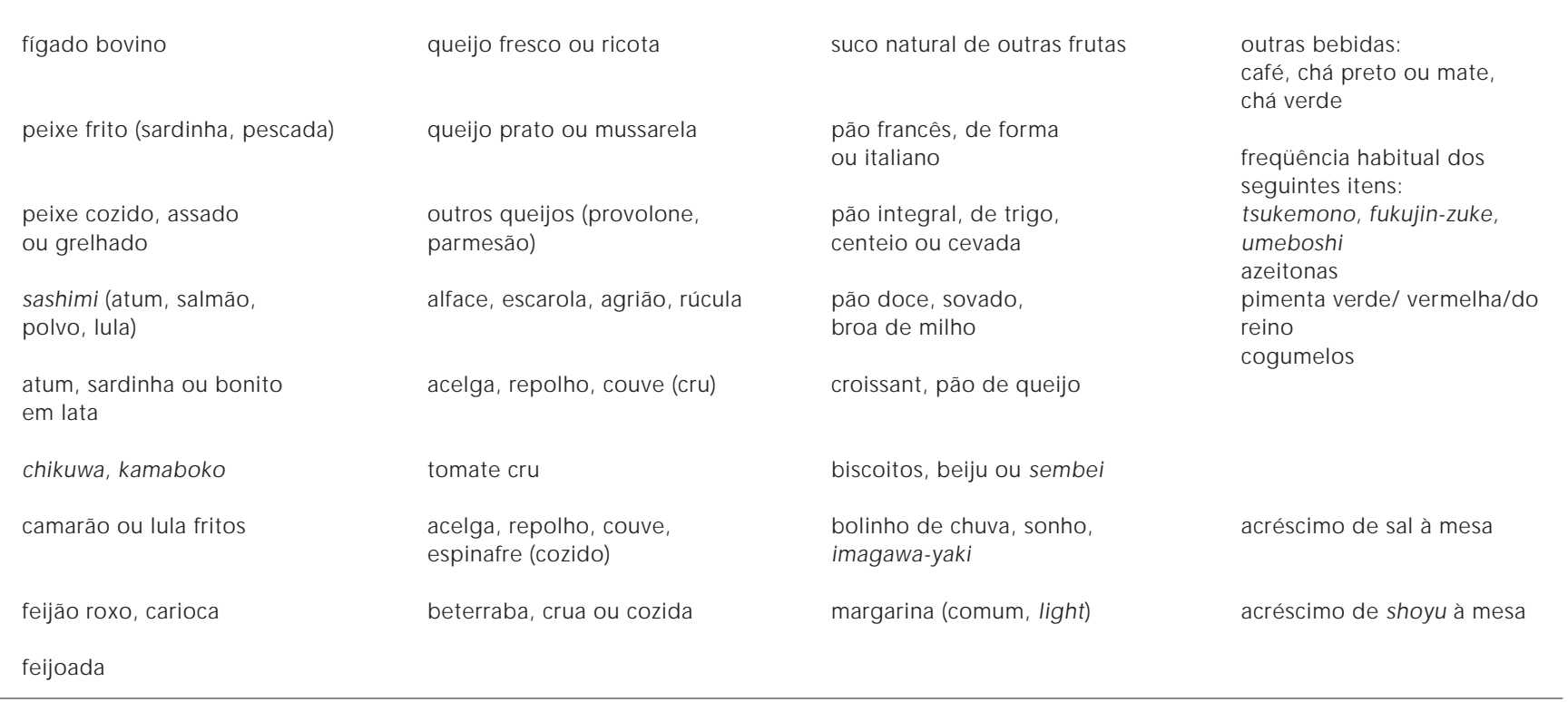

víduos de etnia japonesa ( $n=89$, ambos os sexos e faixa etária entre 20 e 70 anos). Entre os participantes com escolaridade de nível médio ou superior, o QQFA foi auto-administrado sob supervisão. No entanto, o uso de entrevistadores treinados diminuiu a freqüência de erros no preenchimento. $\mathrm{O}$ tempo médio de preenchimento foi de 45 minutos -variação de 30 a 90 minutos -, dependendo da variabilidade da dieta habitual, faixa etária e motivação do participante.

\section{Discussão}

O questionário de freqüência alimentar é instrumento indicado para discriminar a freqüência habitual de consumo alimentar em determinado período de tempo - por exemplo, um mês, seis meses ou um ano. O termo "quantitativo" é utilizado para designar um questionário capaz de estimar também o consumo de nutrientes. Sua utilização em estudos epidemiológicos permite classificar os indivíduos segundo níveis de ingestão para avaliar a associação entre dieta e risco de adoecer. Neste caso, quanto mais discriminativo for - ou seja, capaz de medir a variação de consumo interindividual -, mais adequado será à estimativa de riscos (Thompson \& Byers, 1994).

Para a elaboração deste QQFA, a disponibilidade de dados quantitativos de consumo alimentar possibilitou a seleção inicial de alimentos segundo sua contribuição ao valor calórico total do grupo populacional de interesse. Ado- tou-se também a freqüência de consumo alimentar como variável contínua, para evitar erro de classificação dos indivíduos segundo níveis de ingestão de alimentos ou nutrientes, como sugerido por Tylavsky \& Sharp (1995). Esses procedimentos contribuem para uma avaliação aceitável do teor de vários nutrientes da dieta habitual (Block et al., 1986). No entanto, o grau de exatidão da estimativa de consumo alimentar do QQFA - etapa posterior à elaboração da versão inicial do questionário - deverá ser analisado em estudo de validação capaz de estimar a dieta habitual no período de um ano.

A análise do valor nutricional das dietas individuais obtidas no QQFA pode ser realizada através do programa de computador Dietsys (versão 4.0) - desenvolvido pelo Instituto Nacional de Câncer dos EUA (Block et al., 1994) com dados adicionais de composição química de alimentos compilados das tabelas oficiais do Brasil e do Japão. Os alimentos e receitas típicas confeccionadas em São Paulo estão sendo analisadas quimicamente com o objetivo de serem incorporadas ao banco de dados para análise nutricional das dietas obtidas no QQFA. Um manual de treinamento de entrevistadores com instruções também para autopreenchimento e análise do QQFA está sendo elaborado para apropriada utilização do instrumento em pesquisa epidemiológica. O entrevistador não precisa ser nutricionista, mas recomenda-se que seja treinado no que se refere aos propósitos do QQFA, forma de preenchimento do questionário e tamanho das porções alimentares. 
Distribuição em percentis $(P)$ do tamanho das porções de alimentos de origem japonesa do questionário quantitativo de freqüência alimentar (em medidas caseiras e/ou equivalente em gramas ou mililitros).

\begin{tabular}{|c|c|c|c|c|}
\hline Item alimentar & P25 & P50 & P75 & P100 \\
\hline Misoshiro & $1 / 2$ tigela $(100 \mathrm{~g})$ & 1 tigela $(200 \mathrm{~g})$ & 2 tigelas $(400 \mathrm{~g})$ & 3 tigelas $(600 \mathrm{~g})$ \\
\hline Yakisoba & $1 / 2$ tigela $(100 \mathrm{~g})$ & 1 tigela $(215 g)$ & $300 \mathrm{~g}$ & $500 \mathrm{~g}$ \\
\hline Kare-raisu & $\begin{array}{l}3 \text { colheres } \\
\text { de sopa }(75 g)\end{array}$ & $\begin{array}{l}1 / 2 \text { tigela ou } \\
1 / 2 \text { prato }(100 \mathrm{~g})\end{array}$ & $300 \mathrm{~g}$ & $340 \mathrm{~g}$ \\
\hline Sukiyaki & $\begin{array}{l}2 \text { colheres } \\
\text { de sopa }(60 \mathrm{~g})\end{array}$ & $120 \mathrm{~g}$ & $200 \mathrm{~g}$ & $300 \mathrm{~g}$ \\
\hline Nishime & $\begin{array}{l}2 \text { colheres } \\
\text { de sopa }(60 \mathrm{~g})\end{array}$ & $\begin{array}{l}4 \text { colheres } \\
\text { de sopa }(120 \mathrm{~g})\end{array}$ & $200 \mathrm{~g}$ & $300 \mathrm{~g}$ \\
\hline Sashimi & 4 fatias $(60 \mathrm{~g})$ & $5-6$ fatias $(90 \mathrm{~g})$ & $150 \mathrm{~g}$ & 15 fatias $(225 \mathrm{~g})$ \\
\hline Chikuwa & $40 \mathrm{~g}$ & 4 pedaços $(80 \mathrm{~g})$ & $120 \mathrm{~g}$ & $160 \mathrm{~g}$ \\
\hline Shiruko & $1 / 4$ tigela $(50 \mathrm{~g})$ & $1 / 2$ tigela $(100 \mathrm{~g})$ & 1 tigela $(200 \mathrm{~g})$ & 1,5 tigela $(300 \mathrm{~g})$ \\
\hline Soja (grão) & $\begin{array}{l}2 \text { colheres } \\
\text { de sopa }(30 \mathrm{~g})\end{array}$ & $\begin{array}{l}3 \text { colheres } \\
\text { de sopa }(60 \mathrm{~g})\end{array}$ & $\begin{array}{l}5 \text { colheres de sopa } \\
\text { ou } 1 / 2 \text { tigela }(100 \mathrm{~g})\end{array}$ & 1 tigela $(200 \mathrm{~g})$ \\
\hline $\begin{array}{l}\text { Tofu } \\
\text { (queijo de soja) }\end{array}$ & $\begin{array}{l}1 \text { pedaço } \\
(25 \mathrm{~g})\end{array}$ & $\begin{array}{l}2 \text { pedaços } \\
(50 \mathrm{~g})\end{array}$ & $\begin{array}{l}3 \text { pedaços } \\
(75 \mathrm{~g})\end{array}$ & $\begin{array}{l}5 \text { pedaços } \\
(125 \mathrm{~g})\end{array}$ \\
\hline N atto & $\begin{array}{l}2 \text { colheres } \\
\text { de sopa }(30 \mathrm{~g})\end{array}$ & $\begin{array}{l}3 \text { colheres } \\
\text { de sopa }(60 \mathrm{~g})\end{array}$ & $\begin{array}{l}5 \text { colheres de sopa } \\
\text { ou } 1 / 2 \text { tigela }(100 \mathrm{~g})\end{array}$ & 1 tigela $(200 \mathrm{~g})$ \\
\hline Leite de soja & $100 \mathrm{ml}$ & 1 pacote $(200 \mathrm{ml})$ & $400 \mathrm{ml}$ & $500 \mathrm{ml}$ \\
\hline Miso & $\begin{array}{l}1 \text { colher } \\
\text { de café }(3 g)\end{array}$ & $\begin{array}{l}1 \text { colher } \\
\text { de chá }(5 g)\end{array}$ & $\begin{array}{l}1 \text { colher de } \\
\text { sopa rasa }(10 \mathrm{~g})\end{array}$ & $\begin{array}{l}1 \text { colher de } \\
\text { sopa cheia }(20 \mathrm{~g})\end{array}$ \\
\hline Arroz japonês & $\begin{array}{l}2 \text { colheres } \\
\text { de sopa }(60 \mathrm{~g})\end{array}$ & 1 tigela $(200 \mathrm{~g})$ & $300 \mathrm{~g}$ & 2 tigelas $(400 \mathrm{~g})$ \\
\hline $\begin{array}{l}\text { Sushi, norimaki, } \\
\text { makisushi }\end{array}$ & $\begin{array}{l}2 \text { unidades } \\
\text { ou fatias }(80 \mathrm{~g})\end{array}$ & $\begin{array}{l}3 \text { unidades } \\
\text { ou fatias }(120 \mathrm{~g})\end{array}$ & $\begin{array}{l}4 \text { unidades } \\
(160 \mathrm{~g})\end{array}$ & $\begin{array}{l}5 \text { unidades } \\
(200 \mathrm{~g})\end{array}$ \\
\hline Konnyaku & $\begin{array}{l}1 \text { colher } \\
\text { de sopa }(20 \mathrm{~g})\end{array}$ & $\begin{array}{l}2 \text { colheres } \\
\text { de sopa }(45 \mathrm{~g})\end{array}$ & $\begin{array}{l}3 \text { colheres } \\
\text { de sopa }(65 \mathrm{~g})\end{array}$ & $110 \mathrm{~g}$ \\
\hline Tempura & 2 pedaços $(40 \mathrm{~g})$ & 3 pedaços $(60 \mathrm{~g})$ & $150 \mathrm{~g}$ & $180 \mathrm{~g}$ \\
\hline
\end{tabular}


Figura 1

Questionário quantitativo de freqüência alimentar (Q Q FA). A primeira parte fornece instruções gerais para preenchimento do questionário; a segunda informa o significado das abreviações utilizadas no QQFA; a terceira é exemplo de parte do questionário.

Figura 1a

As questões seguintes relacionam-se ao seu hábito alimentar usual no período de um ano. Para cada quadro, responda, por favor, a freqüência que melhor descreva quantas vezes você costuma comer cada item e a respectiva unidade de tempo (se por dia, por semana, por mês ou no ano). Depois, responda qual a sua porção individual usual em relação à porção média indicada. Escolha somente um círculo para cada coluna.

Muitos grupos de alimentos incluem exemplos. Eles são sugestões e você pode não consumir todos os itens indicados. Se você não come ou raramente come determinado item, preencha o círculo da primeira coluna ( $\mathrm{N}=$ nunca come). $\mathrm{N}$ ão deixe itens em branco.

Figura $1 b$

\begin{tabular}{|c|c|c|c|c|}
\hline \multirow{2}{*}{$\begin{array}{l}\text { Grupos de } \\
\text { alimentos }\end{array}$} & \multicolumn{2}{|c|}{ Com que freqüência você costuma comer? } & \multicolumn{2}{|c|}{ Qual tamanho de sua porção em relação à porção média? } \\
\hline & Quantas vezes você come: & Unidade & Porção média (M) & Sua porção \\
\hline $\begin{array}{l}\text { Alimentos e } \\
\text { preparações }\end{array}$ & $\begin{array}{l}\text { Número de vezes: } \\
1,2,3 \text { etc. ( } \mathrm{N}=\text { nunca } \\
\text { ou raramente comeu } \\
\text { no último ano) }\end{array}$ & $\begin{array}{l}D=\text { por dia } \\
S=\text { por semana } \\
M=\text { por mês } \\
A=\text { por ano }\end{array}$ & $\begin{array}{l}\text { Porção média } \\
\text { de referência }\end{array}$ & $\begin{array}{l}P=\text { menor que a porção média } \\
M=\text { igual à porção média }(M) \\
G=\text { maior que a porção } M \\
E=\text { bem maior que a porção } M\end{array}$ \\
\hline
\end{tabular}

\begin{tabular}{|c|c|c|c|c|c|c|}
\hline \multirow{2}{*}{$\begin{array}{l}\text { Massas } \\
\text { Macarronada, ravióli }\end{array}$} & \multirow{2}{*}{$\begin{array}{l}\text { Quantas vezes você come } \\
\begin{array}{lllllllllll}\text { N } & 1 & 2 & 3 & 4 & 5 & 6 & 7 & 8 & 9 & 10\end{array}\end{array}$} & Unidade & \multirow{2}{*}{$\begin{array}{l}\text { Porção média } \\
1 \text { prato médio }(110 \mathrm{~g})\end{array}$} & \multicolumn{3}{|c|}{ Sua porção } \\
\hline & & $D S M A$ & & $P \quad M$ & G & $\mathrm{E}$ \\
\hline & 00000000000 & 0000 & & 00 & 0 & 0 \\
\hline \multirow[t]{2}{*}{ Lasanha ou caneloni } & $\begin{array}{lllllllllll}N & 1 & 2 & 3 & 4 & 5 & 6 & 7 & 8 & 9 & 10\end{array}$ & $D S M A$ & $1 / 2$ prato médio $(175 \mathrm{~g})$ & $P \quad M$ & G & $E$ \\
\hline & 00000000000 & $\begin{array}{llll}0 & 0 & 0 & 0\end{array}$ & & 00 & 0 & 0 \\
\hline \multirow[t]{2}{*}{ Pizza } & $\begin{array}{lllllllllll}N & 1 & 2 & 3 & 4 & 5 & 6 & 7 & 8 & 9 & 10\end{array}$ & $D S M A$ & 2 fatias médias ou & $P \quad M$ & G & $E$ \\
\hline & 00000000000 & 0000 & 1 brotinho $(220 \mathrm{~g})$ & 00 & 0 & 0 \\
\hline \multirow[t]{2}{*}{ Pastel de feira, Guioza } & $\begin{array}{lllllllllll}N & 1 & 2 & 3 & 4 & 5 & 6 & 7 & 8 & 9 & 10\end{array}$ & $D S M A$ & 1 unidade média ou & $P \quad M$ & G & $E$ \\
\hline & 00000000000 & $\begin{array}{llll}0 & 0 & 0 & 0\end{array}$ & 3 guiozas $(70 \mathrm{~g})$ & & & \\
\hline Pastelaria salgada & $\begin{array}{lllllllllll}N & 1 & 2 & 3 & 4 & 5 & 6 & 7 & 8 & 9 & 10\end{array}$ & $D S M A$ & 1 unidade ou & $P \quad M$ & G & $E$ \\
\hline (torta, empadão, esfiha) & 00000000000 & $\begin{array}{llll}0 & 0 & 0 & 0\end{array}$ & pedaço médio $(80 \mathrm{~g})$ & 00 & 0 & 0 \\
\hline
\end{tabular}

\section{Agradecimentos}

Aos pesquisadores Dr. Gerson Shigueaki Hamada (Centro Nikkei de Prevenção de Doenças, São Paulo) e Dr. Shoichiro Tsugane (Divisão de Epidemiologia, Instituto Nacional de Pesquisa em Câncer, Japão), por permitirem o acesso aos dados dos inquéritos de saúde e nutrição em imigrantes japoneses da cidade de São Paulo. Ao Prof. Dr. José Maria Pacheco de Souza (Faculdade de Saúde Pública, Universidade de São Paulo), pela leitura e sugestões no preparo do manuscrito. Este trabalho foi desenvolvido com auxílio financeiro da Fundação de Amparo à Pesquisa do Estado de São Paulo (FAPESP) e com bolsa de produtividade em pesquisa do Conselho Nacional de Desenvolvimento Científico eTecnológico (CNPq).

\section{Referências}

BENFANTE, R.; YANO, K.; HWANG, L. J.; CURB, J. D.; KAGAN, A. \& ROSS, W., 1994. Elevated serum cholesterol is a risk factor for both coronary heart disease and thromboembolic stroke in Hawaiian Japanese men. Implications of shared risk. Stroke, 25:814-820.

BLOCK, G.; HARTM AN, A. M.; DRESSER, C. M.; CARROLL, M. D.; GANNON, J. \& GARDNER, L., 1986. A data-based approach to diet questionnaire design and testing. American Journal of Epidemiology, 124:453-469.

BLOCK, G.; COYLE, L. M.; HARTMAN, A. M. \& SCOPPA, S. M., 1994. Revision of dietary analysis software for the Health Habits and History Question- 
naire. American Journal of Epidemiology, 139: 1190-1196.

CARDOSO, M. A.; HAM ADA, G. S.; SOUZA, J. M. P.; TSUGANE, S. \& TOKUDOME, S., 1997. Dietary patterns in Japanese migrants to southeastern Brazil and their descendants. Journal of Epidemiology, 7:198-204.

CENTER FOR JAPAN-BRAZIL STUDIES, 1988. The Re sult of Survey for Japanese Population in Brazil. São Paulo: Center for Japan-Brazil Studies.

HANKIN, J. H. \& WILKENS, L., 1994. Development and validation of dietary assessment methods for culturally diverse populations. American Journal of Clinical Nutrition, 59:198S-200S.

HANKIN, J. H., 1986. 23rd Lenna Frances Cooper memorial Lecture: A diet history method for research, clinical, and community use. Journal of American Dietetic Association, 86:868-875.

HAENSZEL, W. \& KURIHARA, M., 1968. Studies of Japanese migrants. I. Mortality from cancer and other diseases among Japanese in the United States. Journal of the National Cancer Institute, 40:43-68.

IBGE (Fundação Instituto Brasileiro de Geografia e Estatística), 1985. Tabela de Composi ção de Alimentos. Rio de Janeiro: IBGE.

LEE, J.; KOLONEL, L. N. \& HANKIN, J. H., 1983. On establishing the interchangeability of different dietary-intake assessment methods used in studies of diet and cancer. Nutrition and Cancer, 5:215218

LIU, K., 1994. Statistical issues related to semiquantitative food-frequency questionnaires. American Journal of Clinical Nutrition, 59:262S-265S.

McGEE, D.; RHOADS, G.; HANKIN, J.; YANO, K. \& TILLOTSON, J., 1982. Within-person variability of nutrient intake in a group of Hawaiian men of Japanese ancestry. American Journal of Clinical Nutrition, 36:657-663.

NELSON, M.; BLACK, A. E.; MORRIS, J. A. \& COLE, T. J., 1989. Between - and within - subject variation in nutrient intake from infancy to old age: Estimating the number of days required to rank dietary intakes with desired precision. American Journal of Clinical Nutrition, 50:155-167.
OLINTO, M. T.; VICTORA, C. G.; BARROS, F. C. \& GIGANTE, D. P., 1995. Twenty-four-hour recall overestimates the dietary intake of malnourished children. Journal of Nutrition, 125:880-884.

RESOURCES COUNCIL, SCIENCE AND TECHNOLOGY AGENCY, 1993. Standard Tables of Food Composition in Japan. 4th Ed. Tokyo: Women's University of Nutrition Press.

THOM PSON, F. E. \& BYERS, T., 1994. Dietary assessment resource manual. Journal of Nutrition, 124:2245S-2317S.

TSUBONO, Y.; TAKAM ORI, S.; KOBAYASHI, M.; TAKAHASHI, T.; IWASE, Y.; LITOI, Y.; AKABANE, M.; YAMAGUCHI, M. \& TSUGANE, S., 1996. A databased approach for designing a semiquantitative food frequency questionnaire for a populationbased prospective study in Japan. Journal of Epidemiology, 6:45-53.

TSUGANE, S.; HAMADA, G. S.; SOUZA, J. M. P.; GOTLIEB, S. L. D.; TAKASHIMA, Y.; TODORIKI, H.; KABUTO, M.; KARITA, K.; YAMAGUCHI, M.; WATANABE, S. \& LAURENTI, R., 1994. Lifestyle and health related factors among randomly selected japanese residents in the city of São Paulo, Brazil, and their comparisons with japanese in Japan. Journal of Epidemiology, 4:37-46.

TYLAVSKY, F. A. \& SHARP, G. B., 1995. Misclassification of nutrient and energy intake from use of closed-ended questions in epidemiologic research. American Journal of Epidemiology, 142: 342-352.

WILLETT, W. C., 1994. Future directions in the development of food-frequency questionnaires. American Journal of Clinical Nutrition, 59:171S-174S.

WILLETT, W. C., 1998. Nutritional Epidemiology. 2nd Ed. Oxford: Oxford University Press.

WILLETT, W. C.; SAMPSON, L.; STAMPFER, M. J.; ROSNER, B.; BAIN, C.; WITSCHI, J.; HENNEKENS, C. H. \& SPEIZER, F. E., 1985. Reproducibility and validity of a semiquantitative food frequency questionnaire. American Journal of Epidemiology, 122:51-65. 